\title{
Sterile Plus Active Neutrinos and Neutrino Oscillations
}

\author{
Leonard S. Kisslinger \\ Department of Physics, Carnegie Mellon University, Pittsburgh, PA 15213 \\ July 26, 2021 \\ PACS Indices:11.30.Er,14.60.Lm,13.15.+g
}

\begin{abstract}
Using a $3+1$ neutrino model with one sterile and the three standard active neutrinos with a $4 \mathrm{x} 4$ unitary transformation matrix, U, relating flavor to mass neutrino states, the probability of $\nu_{\mu}$ to $\nu_{e}$ transition is estimated using sterile-active neutrino masses determined by MiniBooNE and other experiments and sterile-active neutrino angles in the $4 \mathrm{x} 4 \mathrm{U}$ matrix.
\end{abstract}

\section{Introduction}

This is an extension of work on time reversal violation[1] and CP violation[2] via neutrino oscillations. That work used S-matrix theory with a $3 \times 3$ matrix to relate the standard three flavor neutrinos to three neutrinos with well-defined mass. In the present work we extend the standard model by including a fourth neutrino, a sterile neutrino. Recent experiments on neutrino oscillations[3] (see Ref[3] for references to earlier experiments) have suggested the existence of at least one sterile neutrino and the mass used in the present work. See Ref[4] for a discussion of sterile neutrino oscillations and references to experimental and theoretical publications, and Ref[5] for a 6x6 matrix model. Also, pulsar velocities have been estimated using sterile neutrino emission [6] based on a recent estimate[7] of the $\nu_{e}-\nu_{s}$ mixing angle.

Our present work is most closely related to Ref[2] in which CPV was studied. $\mathcal{P}\left(\nu_{a} \rightarrow \nu_{b}\right)$ is the transition probability for a neutrino of flavor $a$ to convert to a neutrino of flavor $b$; and similarly for antineutrinos $\bar{\nu}_{a}, \bar{\nu}_{b}$. The CPV probability difference for $\nu_{\mu}$ to $\nu_{e}$ oscillation is defined as

$$
\Delta \mathcal{P}_{\mu e}^{C P}=\mathcal{P}\left(\nu_{\mu} \rightarrow \nu_{e}\right)-\mathcal{P}\left(\bar{\nu}_{\mu} \rightarrow \bar{\nu}_{e}\right)
$$


A main objective of Ref[2] was to find the dependence of $\Delta \mathcal{P}_{\mu e}^{C P}$ on the parameter $\delta_{C P}$. The antineutrino oscillation probability is related to the neutrino oscillation probability by the neutrino matter potential $V \rightarrow-V$ and $\delta_{C P} \rightarrow-\delta_{C P}$. Since in the present work we are only investigating how $\mathcal{P}\left(\nu_{\mu} \rightarrow \nu_{e}\right)$ is modified by the introduction of a sterile neutrino we set both $V$ and $\delta_{C P}$ equal to zero. $\mathcal{P}\left(\nu_{\mu} \rightarrow \nu_{e}\right)$ is not very dependent on either quantity[2].

\section{$2 \mathcal{P}\left(\nu_{\mu} \rightarrow \nu_{e}\right)$ Derived Using the U Matrix}

This is an exension of the method introduced by Sato and collaborators for three active neutrino oscillations [8, 9] to three active neutrinos plus one sterile neutrino. Active neutrinos with flavors $\nu_{e}, \nu_{\mu}, \nu_{\tau}$ and a sterile neutrino $\nu_{s}$ are related to neutrinos with definite mass by

$$
\nu_{f}=U \nu_{m},
$$

where $U$ is a $4 \times 4$ matrix and $\nu_{f}, \nu_{m}$ are $4 \times 1$ column vectors, which is an extension of the $3 \times 3$ matrix used in Refs. [8, 9] (with $s_{i j}, c_{i j}=\sin \theta_{i j}, \cos \theta_{i j}$ ),

$$
\begin{aligned}
& U=O^{23} \phi O^{13} O^{12} O^{14} O^{24} O^{34} \text { with } \\
& O^{23}=\begin{array}{llll}
1 & 0 & 0 & 0 \\
0 & c_{23} & s_{23} & 0 \\
0 & -s_{23} & c_{23} & 0 \\
0 & 0 & 0 & 1
\end{array} O^{13}=\begin{array}{ccccccc}
c_{13} & 0 & s_{13} & 0 \\
0 & 1 & 0 & 0 \\
-s_{13} & 0 & c_{13} & 0 \\
0 & 0 & 0 & 1
\end{array} O^{12}=\begin{array}{cccc}
c_{12} & s_{12} & 0 & 0 \\
-s_{12} & c_{12} & 0 & 0 \\
0 & 0 & 1 & 0 \\
0 & 0 & 0 & 1
\end{array}
\end{aligned}
$$

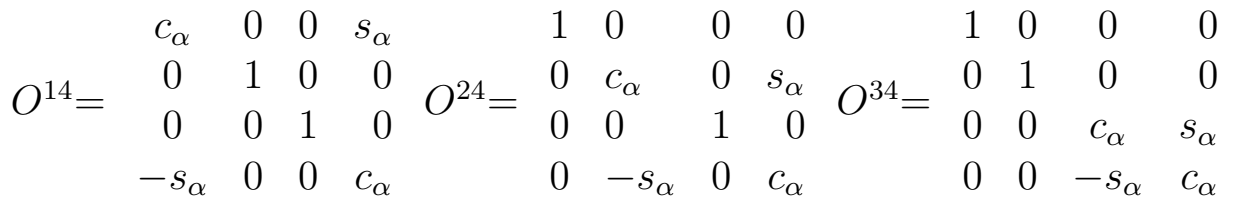

$$
\begin{aligned}
& \begin{array}{llll}
1 & 0 & 0 & 0
\end{array} \\
& \phi=\begin{array}{cccc}
0 & 1 & 0 & 0 \\
0 & 0 & e^{i \delta_{C P}} & 0
\end{array} \\
& \begin{array}{llll}
0 & 0 & 0 & 1
\end{array}
\end{aligned}
$$

with $c_{12}=.83, s_{12}=.56, s_{23}=c_{23}=.7071$. We use $s_{13}=.15$ from the recent Daya Bay Colaboration[10]. In our present work we assume the angles $\theta_{j 4} \equiv \alpha$ for all three $\mathrm{j}$, and $s_{\alpha}, c_{\alpha}=\sin \alpha, \cos \alpha$. An important aspect of our work is to find the dependence of neutrino oscillation probabilities on $s_{\alpha}, c_{\alpha}$.

From $\mathrm{Eq}(3)$ the $4 \times 4 U$ matrix is 


$$
\begin{array}{clcr}
c_{12} c_{13} c_{\alpha} & c_{13}\left(s_{12} c_{\alpha}-c_{12} s_{\alpha}^{2}\right) & -c_{13} s_{\alpha}^{2}\left(c_{12} c_{\alpha}+s_{12}\right)+s_{13} c_{\alpha} & c_{13} s_{\alpha} c_{\alpha}\left(c_{12} c_{\alpha}+s_{12}\right)+s_{13} s_{\alpha} \\
A c_{\alpha} & -A s_{\alpha}^{2}+B c_{\alpha} & -A s_{\alpha}^{2} c_{\alpha}-B s_{\alpha}^{2}+c_{13} s_{23} e^{i \delta_{C P}} c_{\alpha} & A s_{\alpha} c_{\alpha}^{2}+B s_{\alpha} c_{\alpha}+c_{13} s_{23} e^{i \delta_{C P}} s_{\alpha} \\
C c_{\alpha} & -C s_{\alpha}^{2}+D c_{\alpha} & -C s_{\alpha}^{2} c_{\alpha}-D s_{\alpha}^{2}+c_{13} c_{23} e^{i \delta_{C P}} c_{\alpha} & C s_{\alpha} c_{\alpha}^{2}+D s_{\alpha} c_{\alpha}+c_{13} s_{23} e^{i \delta_{C P}} s_{\alpha} \\
-s_{\alpha} & -s_{\alpha} c_{\alpha} & -s_{\alpha} c_{\alpha}^{2} & c_{\alpha}^{3}
\end{array}
$$

with

$$
\begin{aligned}
& A=-\left(c_{23} s_{12}+c_{12} s_{13} s_{23} e^{i \delta_{C P}}\right) \\
& B=\left(c_{23} c_{12}-s_{12} s_{13} s_{23} e^{i \delta_{C P}}\right) \\
& C=\left(s_{23} s_{12}-c_{12} s_{13} c_{23} e^{i \delta_{C P}}\right) \\
& D=-\left(s_{23} c_{12}+s_{12} s_{13} c_{23} e^{i \delta_{C P}}\right) .
\end{aligned}
$$

Using the formalism of Refs. [8, 9] extended to four neutrinos, the transition probability $\mathcal{P}\left(\nu_{\mu} \rightarrow \nu_{e}\right)$ is obtained from the $4 \times 4 \mathrm{U}$ matrix and the neutrino mass differences $\delta m_{i j}^{2}=m_{i}^{2}-m_{j}^{2}$ for a neutrino beam with energy $E$ and baseline $L$ by $[8]$

$$
\mathcal{P}\left(\nu_{\mu} \rightarrow \nu_{e}\right)=\sum_{i=1}^{4} \sum_{j=1}^{4} U_{1 i} U_{1 j}^{*} U_{2 i}^{*} U_{2 j} e^{-i\left(\delta m_{i j}^{2} / E\right) L}
$$

or, since with $\delta_{C P}=0 U_{i j}^{*}=U_{i j}$,

$$
\begin{aligned}
\mathcal{P}\left(\nu_{\mu} \rightarrow \nu_{e}\right)= & U_{11} U_{21}\left[U_{11} U_{21}+U_{12} U_{22} e^{-i \delta L}+U_{13} U_{23} e^{-i \Delta L}+U_{14} U_{24} e^{-i \gamma L}\right]+ \\
& U_{12} U_{22}\left[U_{11} U_{21} e^{-i \delta L}+U_{12} U_{22}+U_{13} U_{23} e^{-i \Delta L}+U_{14} U_{24} e^{-i \gamma L}\right]+ \\
& U_{13} U_{23}\left[U_{11} U_{21} e^{-i \Delta L}+U_{12} U_{22} e^{-i \Delta L}+U_{13} U_{23}+U_{14} U_{24} e^{-i \gamma L}\right]+ \\
& U_{14} U_{24}\left[\left(U_{11} U_{21}+U_{12} U_{22}+U_{13} U_{23}\right) e^{-i \gamma L}+U_{14} U_{24}\right]
\end{aligned}
$$

with $\delta=\delta m_{12}^{2} / 2 E, \Delta=\delta m_{13}^{2} / 2 E, \gamma=\delta m_{j 4}^{2} / 2 E(\mathrm{j}=1,2,3)$. The neutrino mass differences are $\delta m_{12}^{2}=7.6 \times 10^{-5}(\mathrm{eV})^{2}, \delta m_{13}^{2}=2.4 \times 10^{-3}(\mathrm{eV})^{2}$; and we use both $\delta m_{j 4}^{2}=0.9(\mathrm{eV})^{2}$ and $\delta m_{j 4}^{2}=0.043(\mathrm{eV})^{2}$, since $\delta m_{j 4}^{2}=0.043(\mathrm{eV})^{2}$ was the best fit parameter found via the 2013 MiniBooNE analysis, while $\delta m_{j 4}^{2}=0.9(\mathrm{eV})^{2}$ is the best fit using the 2013 MiniBooNE data and previous experimental fits 3 .

Note that in Refs[1, 2] $\mathcal{P}\left(\nu_{\mu} \rightarrow \nu_{e}\right)=\left|S_{12}\right|^{2}$, with $S_{12}$ obtained from the $3 \times 3$ U-matrix and the $\delta m_{i j}$ parameters. Therefore our formalism, given by Eq(15), is quite different, and as will be shown for the same $L, E$ the magnitude of $\mathcal{P}\left(\nu_{\mu} \rightarrow \nu_{e}\right)$ is also different. 
From $\mathrm{Eq}([6)$,

$$
\begin{aligned}
\mathcal{P}\left(\nu_{\mu} \rightarrow \nu_{e}\right)= & U_{11}^{2} U_{21}^{2}+U_{12}^{2} U_{22}^{2}+U_{13}^{2} U_{23}^{2}+ \\
& U_{14}^{2} U_{24}^{2}+2 U_{11} U_{21} U_{12} U_{22} \cos \delta L+ \\
& 2\left(U_{11} U_{21} U_{13} U_{22}+U_{12} U_{22} U_{13} U_{23}\right) \cos \Delta L+ \\
& 2 U_{14} U_{24}\left(U_{11} U_{21}+U_{12} U_{22}+U_{13} U_{23}\right) \cos \gamma L
\end{aligned}
$$

Using the parameters given above,

$$
\begin{aligned}
& U_{11}=.822 c_{\alpha} \quad U_{12}=-.554 s_{\alpha}^{2}+0.084 c_{\alpha} \\
& U_{13}=-.822 s_{\alpha}^{2} c_{\alpha}-.554 s_{\alpha}^{2}+.15 c_{\alpha} \quad U_{14}=.822 s_{\alpha} c_{\alpha}^{2}+.554 s_{\alpha} c_{\alpha}+.15 s_{\alpha} \\
& U_{21}=-.484 c_{\alpha} \quad U_{22}=.484 s_{\alpha}^{2}+.527 c_{\alpha} \\
& U_{23}=.484 c_{\alpha}-.527 s_{\alpha}^{2}+.7 c_{\alpha} \quad U_{24}=-.484 s_{\alpha} c_{\alpha}^{2}+.527 s_{\alpha} c_{\alpha}+.7 s_{\alpha}
\end{aligned}
$$

\section{$2.1 \mathcal{P}\left(\nu_{\mu} \rightarrow \nu_{e}\right)$ for the $3 \times 3$ theory}

First we give the results from using the $3 \times 3$ theory[2] for $\mathcal{P}\left(\nu_{\mu} \rightarrow \nu_{e}\right)$ for comparison with the $4 \times 4$ theory results given in the next subsection. In this previous work a main goal was to study the dependence of $\mathcal{P}\left(\nu_{\mu} \rightarrow \nu_{e}\right)$ on $s_{13}$, but now it has been determined[10] to be approximately 0.15 .

The results using the $3 \times 3$ theory from Ref[2] are shown in Figure 1.

\section{$2.2 \mathcal{P}\left(\nu_{\mu} \rightarrow \nu_{e}\right)$ for the $4 \times 4$ theory}

With the addition of a sterile neutrino, the 4 th neutrino, there are three new angles, $\theta_{14}, \theta_{24}$, and $\theta_{34}$. Our main assumption is that these three angles are the same, $\theta_{j 4}=\alpha$. The angle $\alpha$ is the main parameter that we are studying.

We also use two values for the sterile-active mass differences. The most widely accepted value for $m_{4}^{2}-m_{1}^{2}$ is $0.9(e V)^{2}\left[3\right.$, but we also use $m_{4}^{2}-$ $m_{1}^{2}=.043(\mathrm{eV})^{2}$ from the 2013 MiniBoonE result to test the sensitivity of $\mathcal{P}\left(\nu_{\mu} \rightarrow \nu_{e}\right)$ to the sterile neutrino-active neutrinos mass differences. Since $m_{4}^{2}-m_{1}^{2}>>m_{j}^{2}-m_{i}^{2}$ for $(\mathrm{i}, \mathrm{j})=1,2,3$, we assume that $m_{4}^{2}-m_{j}^{2}=m_{4}^{2}-m_{1}^{2}$.

Figure 2 shows our results for $\mathcal{P}\left(\nu_{\mu} \rightarrow \nu_{e}\right)$ for the four experiments with $m_{4}^{2}-m_{1}^{2}=0.9(e V)^{2}$ and $\alpha=45^{\circ}, 60^{\circ}, 30^{\circ}$. As one can see, $\mathcal{P}\left(\nu_{\mu} \rightarrow \nu_{e}\right)$ is very strongly dependent on $\alpha$.

Next we use $m_{4}^{2}-m_{1}^{2}=0.043(\mathrm{eV})^{2}$, as found in the recent MiniBooNE experiment, to study the effects of $m_{4}^{2}-m_{1}^{2}$ on $\mathcal{P}\left(\nu_{\mu} \rightarrow \nu_{e}\right)$, with $\alpha=$ $45^{\circ}, 30^{\circ}, 60^{\circ}$, as shown in Figure 3 .

Note for $\alpha=0$ (no sterile-active mixing) $U_{14}=0$. Therefore, $\mathcal{P}\left(\nu_{\mu} \rightarrow \nu_{e}\right)$ is a $3 \times 3$ theory; however, we find that $\mathcal{P}\left(\nu_{\mu} \rightarrow \nu_{e}\right)$ is different with the model of Refs. [8, 9], Eq([6), than the theory used in Ref.[2], shown in Figure 1. 

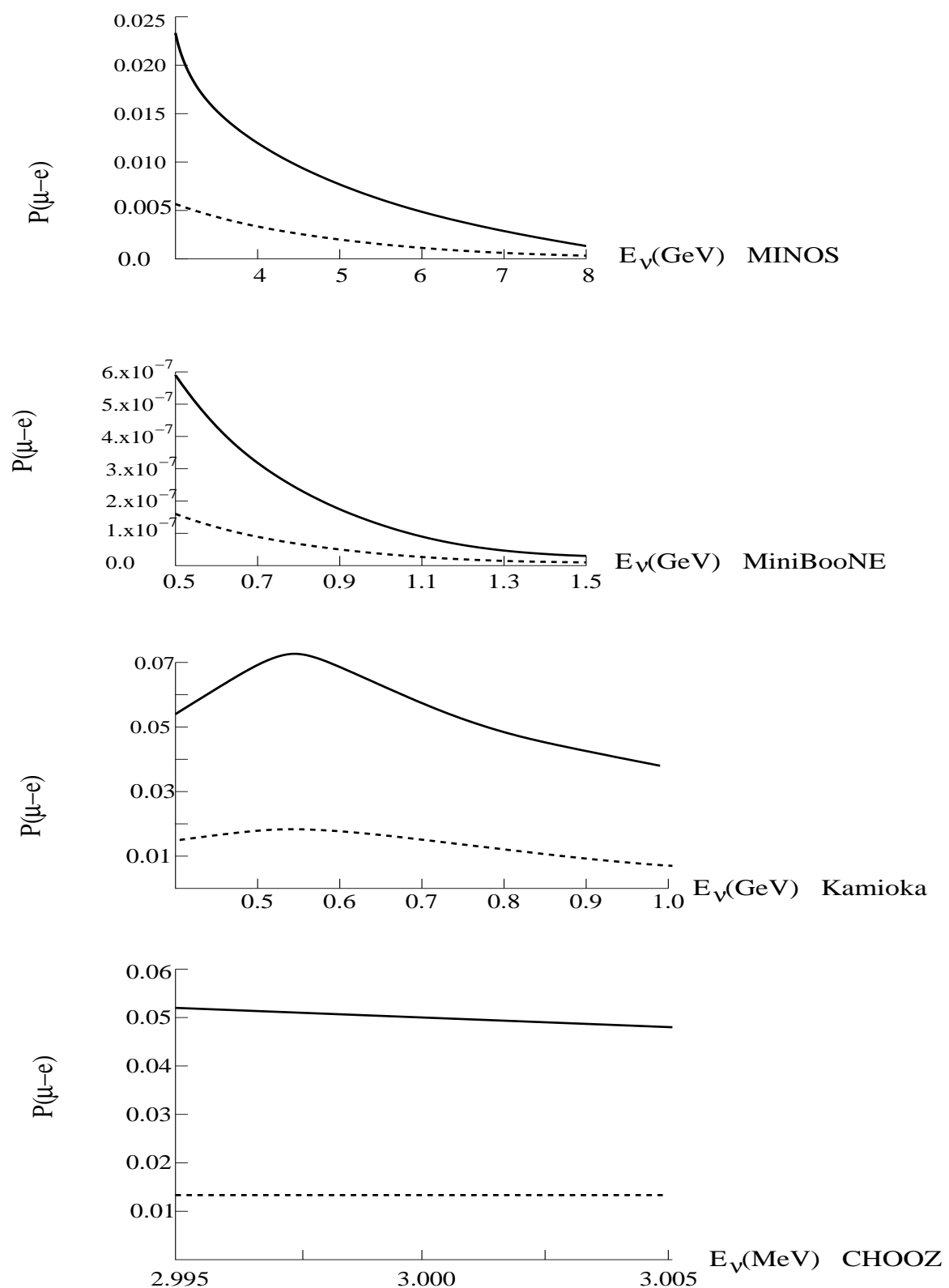

Figure 1: $\quad$ The ordinate is $\mathcal{P}\left(\nu_{\mu} \rightarrow \nu_{e}\right)$ for $\operatorname{MINOS}(\mathrm{L}=735 \mathrm{~km})$, Mini$\operatorname{BooNE}(\mathrm{L}=500 \mathrm{~m})$, JHF-Kamioka $(\mathrm{L}=295 \mathrm{~km})$, and $\mathrm{CHOOZ}(\mathrm{L}=1.03 \mathrm{~km}) \mathrm{us}^{-}$ ing the $3 \times 3 \mathrm{U}$ matrix. Solid curve for $s_{13}=.19$ and dashed curve for $s_{13}=.095$. The curves are almost independent of $\delta_{C P}$. 

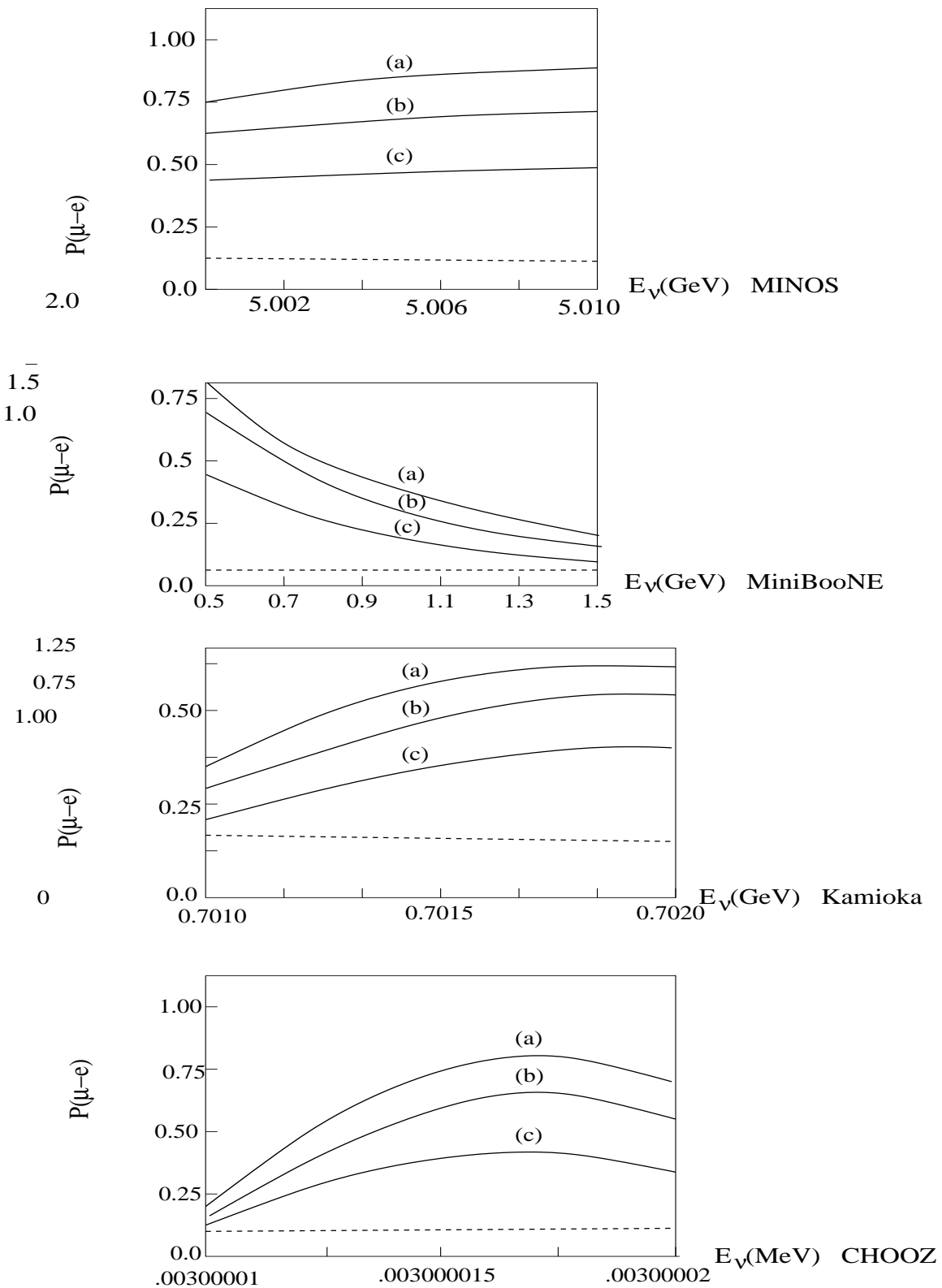

Figure 2: $\quad$ The ordinate is $\mathcal{P}\left(\nu_{\mu} \rightarrow \nu_{e}\right)$ for $\operatorname{MINOS}(\mathrm{L}=735 \mathrm{~km})$, Mini$\operatorname{BooNE}(\mathrm{L}=500 \mathrm{~m})$, JHF-Kamioka $(\mathrm{L}=295 \mathrm{~km})$, and $\mathrm{CHOOZ}(\mathrm{L}=1.03 \mathrm{~km})$ using the $4 \mathrm{x} 4 \mathrm{U}$ matrix with $\delta m_{j 4}^{2}=0.9(e V)^{2}$ and (a),(b),(c) for $\alpha=$ $45^{\circ}, 60^{\circ}, 30^{\circ}$. The dashed curves are for $\alpha=0(3 \times 3)$ 

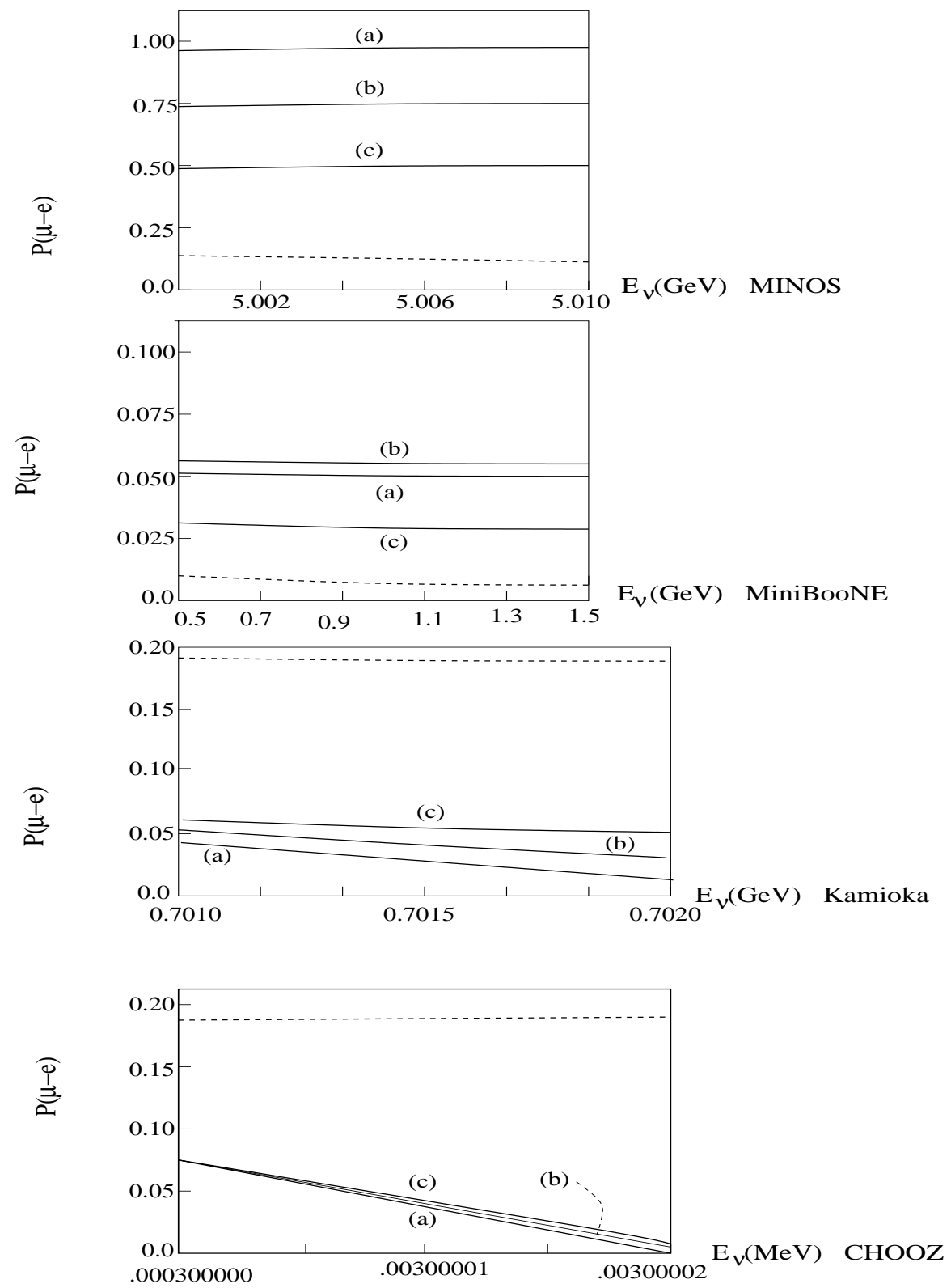

Figure 3: $\quad$ The ordinate is $\mathcal{P}\left(\nu_{\mu} \rightarrow \nu_{e}\right)$ for $\operatorname{MINOS}(\mathrm{L}=735 \mathrm{~km})$, MiniBooNE $(\mathrm{L}=500 \mathrm{~m})$, JHF-Kamioka $(\mathrm{L}=295 \mathrm{~km})$, and $\mathrm{CHOOZ}(\mathrm{L}=1.03 \mathrm{~km})$ using the $4 \times 4 \mathrm{U}$ matrix with $\delta m_{j 4}^{2}=0.043(\mathrm{eV})^{2}$ and (a),(b),(c) for $\alpha=$ $45^{\circ}, 60^{\circ}, 30^{\circ}$. The dashed curves are for $\alpha=0(3 \times 3)$ 


\section{Conclusions}

As shown in the figures, the neutrino oscillation probability, $\mathcal{P}\left(\nu_{\mu} \rightarrow \nu_{e}\right)$, is quite different for a model with four neutrinos, $\nu_{e}, \nu_{\mu}, \nu_{\tau}, \nu_{s}$. $\mathcal{P}\left(\nu_{\mu} \rightarrow \nu_{e}\right)$ is also different for the sterile-active neutrino mass difference $m_{4}^{2}-m_{1}^{2}=$ $0.043(\mathrm{eV})^{2}$ vs $m_{4}^{2}-m_{1}^{2}=0.9(\mathrm{eV})^{2}$, which is favored by experiment.

Our most important result is that $\mathcal{P}\left(\nu_{\mu} \rightarrow \nu_{e}\right)$ is strongly dependent on the sterile-active neutrino mixing angles, with the oscillation probability very different for 30, 60 and 45 degrees for $m_{4}^{2}-m_{1}^{2}=0.9(\mathrm{eV})^{2}$. Therefore, future experiments might be able to determine these sterile-active neutrino parameters. Note that Ref.[7] favors a small $\nu_{e}-\nu_{s}$ mixing angle.

\section{Acknowledgements}

This work was carried out while the author was a visitor at Los Alamos National Laboratory, Group P25. The author thanks Dr. William Louis for information about recent neutrino oscillation experiments, discussions of the theoretical methods used in the present work, and possible future experiments.

\section{References}

[1] E.M. Henley, M.B. Johnson, and L.S. Kisslinger, Int J. Mod. Phys. E 20, $2463(2012)$

[2] L.S. Kisslinger, E.M. Henley, and M.B. Johnson, Int J. Mod. Phys. E 21, 1250065 (2012)

[3] A.A. Aguilar-Arevalo et. al. (MiniBooNE Collaboration), Phys. Rev. Lett. 110, 161801 (2013)

[4] Joachim Kopp et.al., arXiv:1303.3011 (2013)

[5] T. Goldman, Int. J. Mod. Phys. A22, 4967 (2007)

[6] L.S. Kisslinger and M.B. Johnson, Mod. Phys. Lett. 27, 1250215 (2012)

[7] K.N. Abazajian et.al. arXiv:1204.5379 (2012)

[8] Jiro Arafune and Joe Sato, Phys. Rev. D 55, 1653 (1997)

[9] Masafumi Koike and Joe Sato, arXiv:hep-ph/9707203 (1997)

[10] F.P. An et.al., Daya Bay Collaboration, arXiv:1210.6327; Chin. Phys. C37, 01101 (2013) 